\title{
Durable protection of the surface of wood used outdoors: material constraints, problems and approaches to solutions
}

\author{
A. MERLIN ${ }^{1}$, B. GEORGE ${ }^{1}$, L. MALASSENET $^{2}$ and L. PODGORSKI ${ }^{2}$ \\ ${ }^{1}$ Université de Lorraine - LERMAB : Laboratoire d'Etudes et de Recherche sur la Matériau Bois EA 4370 USC INRA, \\ Vandoeuvre les Nancy, France \\ ${ }^{2}$ InstitutTechnologique FCBA: Forêt Cellulose Bois-construction Ameublement, Bordeaux, France
}

\begin{abstract}
The aesthetic durability of wooden structures is a major challenge for the use of this material in construction. Wood is used for its technical performances but also for its architectural qualities and its aesthetic perception. The premature aging of the wooden structures is detrimental because these disorders, even if they do not affect the strength of the structures, are mostly irremediable. The surface protection of wood is generally ensured by the use of a finish, whose essential role is to protect wood from climatic aggressions (water, solar radiation, oxygen, ...). The secondary wood processing industry consists of a series of manufacturing and processing activities, each containing a portion of the added value of the product. The application of a finish on a wood-based work is usually the last and most visible step in this value chain.In outdoor use, the protection of the wood surface with transparent finishes is not yet sufficiently durable to be able to compete with materials used in industrial carpentry such as PVC or aluminum. Opaque finishes generally provide more durable protection but they mask the appearance of the wood sought by users. With the aim of positioning wood in this construction sector, research on transparent finishes has focused on the efficiency and improvement of the durability of the protection of the surface appearance of structures. Faced with climatic aggressions, the optimum conservation of a structure is not only linked to the performance of the finish but also to the characteristics of the wood material. In particular, in order to fulfill its protective function, the finish film must be able to follow the dimensional variations of the wood it covers without breaking and without detachment. In addition to the criteria for the effectiveness of finishes in the protection of structures, the environmental impact must be considered with increasing attention. Currently, more than $80 \%$ of composite or solid wood products are still protected with solvent-based liquid products, which are an important source of VOC emissions. Does the solution come from photo-polymerizable systems that can be formulated with $100 \%$ dry matter either in liquid phase in reactive diluents or powdered?
\end{abstract}

Keywords: Wood, Durability of surface appearance, Surface coatings, Photopolymerizable finishes

\section{Introduction}

Le bois a toujours été un matériau indispensable pour l'homme; avec la terre c'est le matériau le plus ancien qui ait été mis en œuvre. Ses utilisations ont été variées et diversifiées au cours du temps que ce soit pour la fabrication d'objets, comme source d'énergie ou encore dans la construction. L'utilisation du bois en construction remonte à des temps ancestraux. Dès le moyen-âge, avec la forte fréquence des incendies dans les villes, le bois sera de plus en plus remplacé par la pierre pour les constructions urbaines. Au $\mathrm{XIX}^{\circ}$ siècle, avec l'apparition $\mathrm{du}$ béton et la déforestation, les maisons en bois vont pratiquement disparaître dans les pays du sud de l'Europe. Ce n'est pas le cas du nord de l'Europe, des
Etats Unis et du Canada où la couverture forestière est très importante. Ainsi, en Amérique du Nord et en Scandinavie, le bois est employé en structure dans plus de 90\% des maisons individuelles. En France, depuis quelques années, on constate une progression de la part du bois dans le bâtiment à la suite de la crise de l'énergie et des matières premières non renouvelables. Cette évolution est liée à la qualité environnementale du bois et à de nouvelles réglementations thermiques qui peuvent être respectées dans des conditions économiques compétitives en associant le bois à des matériaux isolants.

$\mathrm{Si}$ de nombreux arguments plaident en faveur de l'utilisation du bois en construction :

- $\quad$ sa ressource abondante et renouvelable, 
- son faible coût énergétique,

- sa présentation sous multiples formes couvrant l'ensemble des utilisations potentielles,

- son rapport poids / résistance favorable pour assurer une construction légère,

- sa capacité isolante (12 fois plus isolant que le béton),

- sa résistance au feu,

- son esthétisme,

il présente quelques faiblesses liées à son caractère naturel :

- $\quad$ son anisotropie et son hétérogénéité

- sa sensibilité à l'humidité et son instabilité dimensionnelle

- $\quad$ sa sensibilité aux agents biologiques, physicochimiques et climatiques

duPour pouvoir concurrencer d'autres matériaux utilisés en construction comme les polymères synthétiques, il faudra soit traiter le bois soit le modifier pour améliorer sa durabilité. En effet, face aux matériaux concurrentiels comme le PVC ou l'aluminium, un handicap dont pâlit encore le bois réside dans la durée de vie trop faible de ses finitions et la nécessité de rénovations fréquentes. $\mathrm{Si}$ la garantie décennale, tant du point de vue durabilité du bois que du point de vue de la fonctionnalité de l'ouvrage ne pose pas de problème, l'impossibilité d'assurer en finition, surtout lorsque les produits sont transparents, une garantie d'aspect de surface pénalise considérablement la menuiserie extérieure en bois. Le vieillissement naturel du bois résulte d'une combinaison complexe de divers phénomènes physiques, chimiques et biologiques qui modifient la surface du matériau. En pratique le bois perd sa couleur d'origine et grisaille progressivement. Ce grisaillement s'accompagne d'une érosion très lente, de l'ordre de quelques millimètres par siècle, des couches superficielles du bois. C'est un phénomène purement visuel qui n'entraine aucune dégradation d'ordre mécanique. Cette modification d'aspect de surface est essentiellement due à l'action du rayonnement UV de la lumière solaire, au lessivage de la surface de l'ouvrage et à l'augmentation de la rugosité de surface liée à la dégradation plus importante du bois initial. Parallèlement à cette évolution d'aspect, le bois en ambiance extérieure est soumis à de brusques changements d'humidité dus aux variations climatiques. $\mathrm{Si}$ le bois n'est pas protégé, ces variations rapides d'humidité créent des tensions importantes dans les ouvrages et sont à l'origine de variations dimensionnelles, déformations, fentes, ... L'application d'une finition a donc deux objectifs essentiels : protéger le bois du rayonnement solaire et limiter les variations dimensionnelles du bois en réduisant les échanges entre le bois et l'atmosphère. Il ne faut pas confondre finition et préservation. La finition constitue uniquement une protection de la surface du bois. Elle ne confère pas de protection contre les insectes et les champignons à une essence non durable naturellement et ne peut en aucun cas se substituer à l'opération de préservation dont le but est de rendre durable biologiquement un bois qui ne l'est pas naturellement pour l'emploi auquel on le destine. L'industrie de la deuxième transformation du bois est composée d'une chaîne successive d'activités de fabrication et de transformation qui contiennent chacune une partie de la valeur ajoutée du produit. L'application d'une finition est généralement la dernière étape et la plus visible dans cette chaîne de valeur ajoutée. En plus de l'aspect décoratif, de la protection contre les agressions et de la limitation des reprises d'humidité du bois, des fonctions additionnelles peuvent être apportées : facilité de nettoyage, propriétés auto-nettoyantes, antisalissures, propriétés optiques, effets barrière... On parle alors de finition «intelligente ».

\section{Les facteurs affectant les performances des systèmes bois- finitions}

Les performances des finitions sont tributaires de facteurs liés au bois brut avant l'application de la finition, de facteurs climatiques et de facteurs qui affectent la finition elle-même.

\subsection{Les facteurs liés au bois.}

Les propriétés de surface sont déterminées par l'anatomie et la composition chimique de l'essence de bois. Les cernes annuels et leurs propriétés affectent la façon avec laquelle une finition va être appliquée et la durée de vie de cette finition. En effet un bois va être classé comme plus ou moins facile à revêtir selon la grosseur des cernes et la transition entre les cernes du bois initial et du bois final. Par exemple, le peuplier, le hêtre, l'érable ou le bouleau sont des feuillus dont les cernes présentent des pores de taille et de répartition relativement uniformes. Ces bois ont une texture fine ce qui les rend facile à revêtir. Par contre, le chêne est un feuillu caractérisé par des pores du bois initial plus gros que les pores du bois final ce qui nécessite une préparation de la surface avant l'application d'une finition. Les extractibles, qui représentent $12 \%$ de la masse sèche du bois pour les essences résineuses et jusqu'à $20 \%$ pour des essences tropicales sont responsables de l'odeur, la couleur naturelle ou la résistance aux agents de dégradation biologique. Les extractibles changent de couleur lorsqu'ils sont exposés au rayonnement solaire. Cette dégradation par la lumière des extractibles n'est pas empêchée par un revêtement de surface transparent. Les extractibles peuvent aussi affecter la finition elle-même en retardant son durcissement. Par ailleurs, les nœuds des résineux peuvent exsuder de la résine ce qui confère une fragilité à la finition pouvant aller jusqu'à la rupture du film. L'état de surface après usinage a également une influence sur l'accrochage d'une finition et donc sur la durabilité de la protection.

\subsection{Les facteurs climatiques}

L'impact climatique affecte différemment les composants $\mathrm{du}$ bois que sont les carbohydrates (cellulose, hemicelluloses), les lignines et les extractibles. La lumière solaire est constituée des rayonnements ultraviolets, visibles et infrarouges. Les rayonnements UV qui ne représentent qu'une faible proportion du 
rayonnement solaire sont les plus impliqués dans les réactions de photodégradation du bois qui induisent des altérations de l'aspect de surface des ouvrages. Ce sont des chromophores présents dans la structure moléculaire des lignines et des extractibles qui absorbent la lumière solaire et créent des espèces radicalaires qui sont le point de départ des réactions de photodégradation oxydative. $\mathrm{Si}$ les carbohydrates sont des polymères résistants aux UV, ils sont très sensibles à l'humidité. Les cycles d'humidification du bois puis séchage provoquent des variations dimensionnelles du bois, on dit que le bois travaille. L'eau aura également un impact sur la performance de la finition en agissant sur les liants présents dans les formulations qui sont souvent hydrolysables ce qui peut provoquer une perte des propriétés mécaniques du film. Pour un bois revêtu d'une finition, les variations d'humidité dépendent de la perméabilité (à l'eau liquide et vapeur) du film de finition .En rendant le système imperméable à l'eau, la finition empêche le développement de moisissures.

\subsection{Les facteurs liés à la finition}

La pigmentation a une influence sur la durabilité de la finition aux intempéries. Les pigments apportent la couleur en absorbant ou en réfléchissant les rayonnements UV-visible du rayonnement solaire, ce qui limite la photodégradation des liants. Ainsi pour un même liant, un revêtement pigmenté a une durabilité deux à quatre fois supérieure à celle d'un revêtement transparent. Toutefois, en se dégradant, le liant va permettre aux pigments de se libérer et provoquer le farinage de la finition. Ce farinage se produit avec l'action conjuguée des UV, de l'oxygène et de l'eau. Même si les revêtements pigmentés sont plus durables pour la protection des ouvrages en bois, l'intérêt se porte plus sur les revêtements transparents qui laissent visible la texture du bois mais pour lesquels les performances en extérieur sont à améliorer. Pour les revêtements de surface réticulant par un mécanisme radicalaire, l'oxygène réduit non seulement la vitesse de séchage mais affecte également les propriétés mécaniques des films séchés à l'air. La perméabilité à l'eau liquide et à la vapeur est un paramètre important qui fait l'objet des normes EN 927-5 et ISO 7783. La souplesse de la finition est un paramètre clé pour la durabilité du revêtement appliqué sur le bois. Le manque de souplesse ne va pas permettre au film de s'adapter aux variations dimensionnelles du bois ce qui va entrainer des craquèlements. Le manque d'adhérence qui peut provenir de l'essence de bois ou de la formulation de la finition provoque un écaillage du revêtement de surface.

\section{Les finitions à séchage UV pour le bois : une solution pour la limitation des composés organiques volatils (COV)?}

Les recherches sur les finitions appliquées sur les ouvrages en bois destinés à un emploi intérieur ou extérieur ont essentiellement porté sur l'efficacité et sur la durabilité de la protection. S'il est vrai que ces deux paramètres peuvent conditionner la survie de l'utilisation $\mathrm{du}$ bois en menuiserie industrielle, la prise en compte des facteurs environnementaux doit être considérée avec une attention croissante. Pour se mettre en conformité ou pour devancer une législation de plus en plus contraignante sur la limitation des émissions de COV et pour satisfaire une demande des utilisateurs de plus en plus soucieux des aspects hygiène, santé et environnement lors de l'élaboration et de la durée de vie des matériaux, les formulations des résines de finition pour le bois doivent être reconsidérées dans leur ensemble. Les finitions de demain devront allier performance et respect des contraintes environnementales sans pour autant alourdir le prix de la menuiserie. La diminution des COV dans un atelier de finition peut être atteinte par des actions portant sur les procédés d'application, sur les procédés de nettoyage des appareillages et surtout sur les formulations en favorisant des systèmes à faible taux d'émission des COV. Le durcissement sous rayonnement UV permet l'utilisation de formulations à $100 \%$ de matière sèche par dilution des oligomères dans des diluants réactifs (monomères multifonctionnels photopolymérisables) qui ne s'évaporent pas et qui s'incorporent dans le film en participant à la réticulation. L'ajout de ces diluants permet de diminuer la viscosité des formulations et ainsi de les rendre compatibles avec les procédés d'application. $\mathrm{Ce}$ type de formulations séduisantes au niveau environnemental présente des inconvénients quant aux propriétés mécaniques du film durci. Les diluants réactifs conduisent à des films peu flexibles qui ne conviennent pas à la protection de supports instables dimensionnellement comme le bois. Pour de tels matériaux, la mise au point de formulations photosensibles sans diluant réactif serait une solution et trois voies de recherche sont explorées :

- revêtements liquides à $100 \%$ de matière sèche à base d'oligomères de faible viscosité,

- revêtements UV aqueux. Pour ces systèmes, il faut évaporer l'eau avant la réticulation,

- revêtements «poudre »: l'oligomère est un solide pulvérulent à température ambiante qui, par chauffage, fond en formant un film qui est réticulé par exposition aux UV.

A l'heure actuelle, les finitions à réticulation sous rayonnement UV sont industriellement appliquées sur des ouvrages en bois destinés à des utilisations intérieures (ameublement, parquets, panneaux décoratifs...) et uniquement en finition transparente ou peu pigmentée. Le développement des vernis photoréticulables dans ce secteur est attribué à certains paramètres technologiques et écologiques :

- grande souplesse des caractéristiques physiques des vernis avant et après l'application,

- faible toxicité due à l'absence de solvant,

- grande stabilité thermique des formulations photoréticulables, 
- vitesse de réticulation élevée à température ambiante,

- $\quad$ surface plus faible des installations avec économie d'énergie et d'investissement.

Si l'utilisation des résines photosensibles présente un intérêt au niveau environnemental, elle n'est pas sans poser des problèmes qui ne sont pas résolus, à l'heure actuelle, de façon définitive.

\section{La recherche de solutions pour améliorer la durabilité en utilisation extérieure des systèmes bois-finition photoréticulable transparente}

On peut distinguer des difficultés liées d'une part à la nature photochimique de l'amorçage et d'autre part à la nature du bois.

\subsection{Difficultés liées à l'amorçage}

Actuellement le séchage par un rayonnement UV ne peut être réalisé qu'en atelier de fabrication. De ce fait, l'utilisation des finitions photoréticulables pour la protection des menuiseries extérieures est délicate car elle nécessite la pose sur site de l'ouvrage préalablement revêtu en atelier. L'impossibilité d'appliquer ces finitions sur site pose également le problème de la rénovation. Pour lever ce verrou lié à la nécessité d'utiliser le rayonnement UV, des recherches récentes s'orientent vers le développement de formulations réticulant sous la lumière naturelle dans des conditions adaptées pour le séchage d'un revêtement de surface appliqué sur du bois. Pour obtenir une finition régulière, il faut assurer un flux photonique homogène sur l'ensemble de la pièce à vernir. De ce fait, la finition d'éléments assemblés est techniquement difficile. Avec les amorceurs photochimiques courants dont l'efficacité est maximale dans le domaine UV, le durcissement des finitions pigmentées ne peut être obtenu que sur des faibles épaisseurs. La mise au point de systèmes amorceurs complexes fonctionnant dans les fenêtres de transmission des pigments ont permis le séchage d'un film pigmenté de $100 \square \mathrm{m}$ sur le bois.

\subsection{Difficultés liées à la nature du bois}

Les finitions à séchage UV ont fait l'objet de recherches poussées dans le domaine de la protection des surfaces métalliques utilisées en extérieur, en particulier pour le traitement des carrosseries automobiles. Des formulations très performantes quant à leur tenue sont disponibles sur le marché. Appliquées sur le bois, ces mêmes formulations optimisées pour le traitement des surfaces métalliques n'assurent plus une durabilité de la protection suffisante pour concurrencer des menuiseries en PVC ou en aluminium. Cette perte d'efficacité doit être imputée à des caractéristiques propres du matériau bois.

\section{4,2.1 Le bois est un matériau poreux :}

La pénétration du rayonnement proche UV dans le bois est de l'ordre de $70 \square \mathrm{m}$. En conséquence, la fraction de la résine qui migre plus en profondeur dans la structure poreuse du bois ne sera pas atteinte par les photons nécessaires à l'amorçage. Pour certaines essences poreuses, cette perte de résine peut devenir importante et ainsi alourdir le prix du traitement de surface. L'augmentation de la viscosité de la formulation pour limiter la migration peut devenir incompatible d'une part avec le procédé d'application et d'autre part avec les propriétés mécaniques du film durci.

\section{4,2.2 Le bois est instable dimensionnellement:}

Lors d'un vieillissement climatique de systèmes bois/finition photoréticulable, les dégradations n'ont pas pour origine une perte de cohésion du film de finition. La tenue face aux agressions physico-chimiques de l'environnement d'un revêtement de surface est garantie par l'efficacité des additifs de stabilisation (absorbeurs UV, antioxydants...) entrant dans les formulations des résines pour extérieur. La dégradation se manifeste par une fracture à l'interface bois/finition provoquant un décollement du film. En ambiance extérieure, le bois subit des variations dimensionnelles importantes liées aux variations d'humidité. Pour remplir sa fonction de protection de surface, la finition doit être capable de suivre sans rupture et sans décollement les variations dimensionnelles de l'ouvrage qu'elle recouvre. Différentes stratégies ont été explorées pour améliorer la durabilité de la protection :

- formuler des formulations souples : la flexibilité $\mathrm{du}$ film de finition et la conservation de cette flexibilité durant la période d'utilisation de l'ouvrage sont des paramètres influents sur la rupture à l'interface bois/finition. La flexibilité est obtenue en abaissant la température de transition vitreuse $\mathrm{T}_{\mathrm{g}} \mathrm{du}$ film durci en dessous de la température d'utilisation. Dans le cas des revêtements liquides photosensibles, l'ajout de diluants réactifs pour ajuster la viscosité conduit à des degrés de réticulation trop importants pour pouvoir abaisser suffisamment la $T_{g}$. L'application de revêtements UV aqueux pourrait paraître séduisante; ces résines peuvent être formulées à faible viscosité tout en conduisant à des films flexibles. Toutefois, l'application de ces formulations aqueuses sur le bois se heurte au problème de l'élimination de l'eau nécessaire à la réticulation qui se fait essentiellement par migration dans la structure poreuse du bois. De ce fait, la résine réticule sur une surface gonflée par l'eau. Un passage sous microonde pour éliminer l'eau avant l'excitation UV donne des résultats intéressants. Ce procédé est industrialisé pour la menuiserie 
intérieure (technologie MOS Gardina). De plus, l'eau d'une part favorise la migration en surface de substances extractibles colorées qui provoque des taches peu esthétiques et d'autre part provoque un relevage des fibres qui modifie l'aspect de surface et le toucher des ouvrages.

- Assurer la stabilité dimensionnelle du bois avant l'application de la finition: des traitements thermiques ou chimiques permettent de limiter de façon durable les variations dimensionnelles du bois soumis aux contraintes physico-chimiques de l'environnement. Ces traitements modifient l'énergie de surface et diminuent l'accrochage des revêtements de surface.

Pour corréler ces deux approches, nous avons proposé d'intercaler entre le bois et le film de finition un composite de surface (sous-couche) qui, par ses caractéristiques de flexibilité amortit les variations dimensionnelles du bois et ainsi réduit les sollicitations $\mathrm{du}$ film de finition. Les résultats obtenus sur des formulations simplifiées lors d'un vieillissement accéléré d'échantillons de différentes essences ont montré la faisabilité et l'intérêt de ce procédé qui n'est pas appliqué industriellement jusqu'ici. La durabilité de la protection dépend de la flexibilité du composite de surface et de l'adhérence de la couche de finition sur le composite.

\section{4,2.3 Le bois est un matériau réactif :}

Il est connu des applicateurs que le séchage des finitions alkydes ou polyesters qui réticulent par voie radicalaire est retardé sur les essences riches en substances extractibles caractérisées par une forte teneur en composés phénoliques. Une étude fondamentale de l'influence des molécules phénoliques sur les caractéristiques d'une réaction de réticulation amorcée par voie photochimique nous a montré que la vitesse de séchage et les propriétés mécaniques du film durci dépendent du mode de fonctionnement de l'amorceur et de la réactivité des molécules phénoliques du bois sur les espèces transitoires et radicalaires intervenant dans le mécanisme de polymérisation.

\section{4,2.4 Le bois ne présente pas une pérennité de son aspect de surface :}

Même revêtu d'une finition transparente, l'aspect d'un bois n'est pas immuable et il est susceptible de varier sous l'action de facteurs liés à l'environnement. Le vieillissement de systèmes bois/finition transparente a mis en évidence le rôle protecteur limité des vernis. Les effets principaux du revêtement transparent sont de filtrer une partie du rayonnement proche UV, de faire barrière à l'eau et à l'oxygène. Ainsi, il a pu être montré qu'un bois recouvert d'une finition transparente subit qualitativement les mêmes altérations de l'aspect de surface que le bois brut exposé sous atmosphère d'azote, à la partie visible du rayonnement solaire. De ce fait, la conservation optimale d'un système bois/finition transparente n'est pas seulement tributaire des performances en terme de stabilité de la finition ellemême mais également de la durabilité de l'aspect de surface du bois qu'elle recouvre. Pour proposer un système de finition assurant une pérennité de l'aspect d'un ouvrage en bois qui puisse concurrencer l'utilisation du PVC ou de l'aluminium en menuiserie, il est nécessaire de stabiliser la couleur du bois par un traitement de surface avant l'application de la finition transparente.

\section{Les finitions "poudre» pour la protection du bois}

Le développement de produits de finition en phase aqueuse ou à haut extrait sec contribue à diminuer les émissions de COV mais ces produits ne répondent pas toujours aux attentes du marché quant à la qualité du film de finition. De plus, leur utilisation est fortement consommatrice d'eau et génère de nombreux déchets solides et liquides. L'arrivée sur le marché des résines thermodurcissables a fait évoluer la technique des finitions poudre qui sont actuellement largement utilisées dans l'industrie de la métallurgie. Les avantages des finitions poudre sont nombreux: compatibilité avec l'environnement, facilité d'application, rapidité de changement de couleur, faible consommation d'énergie, récupération facile par aspiration de l'excédent de poudre, réduction des risques sanitaires... Cependant, ce procédé reste peu développé pour les substrats comme le bois. En effet, les formulations poudre utilisées pour la protection des surfaces métalliques ne sont pas applicables sur des matériaux thermosensibles ; la fusion nécessaire pour la formation du film et la réticulation exigent des températures et des temps de chauffe incompatibles avec le bois. C'est avec l'apparition récente de produits polymérisant à basse température ou sous rayonnement UV qu'une application sur le bois est rendue possible. Actuellement, les finitions «poudre» sont uniquement utilisées pour la protection intérieure d'ouvrages en bois ou en matériaux dérivés. Pour les menuiseries extérieures, les formulations commercialisées conduisent à des films pas suffisamment flexibles pour suivre les variations dimensionnelles du support. L'optimisation du procédé et des formulations est un enjeu crucial pour le développement et la promotion d'une telle technologie dans l'industrie du bois.

\section{Conclusion}

La finition est l'étape la plus importante pour ajouter de la valeur aux ouvrages en bois et protéger aussi efficacement que possible leur aspect de surface. La 
durée de vie d'un produit en bois est souvent associée à la durabilité du revêtement de finition. Les progrès les plus marquants dans les finitions transparentes ont été observés en ameublement intérieur; des formulations performantes alliant durabilité de la protection et respect de l'environnement ont pu être mises au point. La durabilité de la protection des ouvrages en extérieur et en revêtement transparent reste insuffisante pour assurer une concurrence efficace de la menuiserie bois face aux polymères synthétiques. Alors que le matériau bois offre en construction des avantages recherchés par les particuliers, les performances des revêtements de surface restent un des freins au développement de la construction en bois. En plus des propriétés de protection de la surface des ouvrages et pour satisfaire les attentes et les exigences de la clientèle, il devient nécessaire de proposer des finitions dites intelligentes, qui auront la capacité de défendre activement et durablement le bienêtre, la santé, le confort... Le développement de ces finitions innovantes représente un saut technologique important dans le domaine de la finition des matériaux en général et du matériau bois en particulier et contribue au développement de produits à plus haute valeur ajoutée. Certaines techniques font déjà l'objet de brevets, mais très peu sont destinées à une application sur du bois. En ce qui concerne l'industrie du bois, le panel des différentes fonctions envisagées est important: parfum, désodorisant, antibactérien, nettoyage facile, absorption des COV, modification de la couleur en fonction de la température, ignifugation, haute résistance thermique (jusqu'à $450^{\circ} \mathrm{C}$ ), préservation biocide, anti microbien, haute conductivité électrique (résistivité inférieure à $10^{4} \Omega$ ), forte isolation électrique (résistivité supérieure à $10^{13} \Omega$ ), résistance chimique particulière (résiste aux hydrocarbures, acides, bases), anti dégazage (pour des supports difficiles à traiter), flexibilité (film postformable)....

\section{Références :}

1. R.A. Williams, M.T. Knaebe, W.C. Feist, Finishes for Exterior Wood: selection, application and maintenance, Forest Products Laboratory (1996)

2. R.J. Tichy, Interior Wood Finishing, Forest Products Laboratory (1997)
3. P. Dulbecco, D. Luro, L'essentiel sur le bois, Document CTBA (1998)

4. M.L. Roux, Finition des ouvrages en bois dans le bâtiment, Document CTBA (1994)

5. Association des Ingénieurs de l'Ecole Supérieure du Bois, Manuel de l'ingénierie Bois, Eyrolles (2013)

6. L. Podgorski, A. Merlin, Systèmes bois-finitions extérieures. Vieillissement, comportement. In : DIROL Daniel, DEGLISE Xavier. Durabilité des bois. Paris: Hermès Science Publication, (2001)

7. Y. Irmouli, Etude de systèmes de finitions pour bois : application de nouvelles formulations, vieillissement et amélioration des performances. Thèse Sciences du bois et des fibres, Université de Lorraine (2007)

8. J. Bessière: Les finitions poudres appliquées sur le bois et matériaux dérivés : optimisation du procédé industriel, étude des relations finition/substrat et fonctionnalisation. Thèse Sciences du bois et des fibres, Université de Lorraine (2012)

9. M. Sylla, Rôle des molécules phénoliques dans la photopolymérisation : application à des résines de finition pour le bois. Thèse Sciences $\mathrm{du}$ bois et des fibres, Université de Lorraine (2001)

10. F. Aloui, Rôle des absorbeurs UV inorganiques sur la photostabilisation des systèmes boisfinition transparente. Thèse Sciences du bois et des fibres, Université de Lorraine (2006)

11. F. Mauguière-Guyonnet, Photopolymérisation radicalaire sous lumière visible: application aux revêtements pour bois. Thèse de l'Université de Haute Alsace, Chimie des matériaux (2004)

12. V. Maurin, Revêtements poudres $U V$ mécanismes de polymérisation et étude des relations structure/propriétés. Thèse de l'Université de Haute Alsace, Chimie des matériaux (2012). 